\title{
Angiomyolipoma with Intraluminal Tumor Thrombus into the Inferior Vena Cava: Insights and Treatment Alternatives
}

\author{
Maria A Ocampo ${ }^{*}$, Cesar Capera², Maria Camila Gonzalez ${ }^{1}$, Javier M Salgado ${ }^{1}$ and Wilfredo Donoso ${ }^{1,3}$ \\ ${ }^{1}$ Division of Urology, Hospital Universitario Mayor Méderi, Universidad del Rosario, Bogotá, Colombia \\ ${ }^{2}$ Division of Urology, Universidad Nacional de Colombia, Colombia \\ ${ }^{3}$ Division of Urology, Hospital Universitario Mayor Méderi, Universidad Nacional de Colombia, associate Universidad del Rosario, Bogotá, Colombia
}

*Corresponding author: Maria A Ocampo, Division of Urology, Hospital Universitario Mayor Méderi, Universidad del Rosario, Bogotá, Colombia, E-mail: mariaan.ocampo@urosario.edu.co

Received: 18 Jan, 2021 | Accepted: 25 Jan, 2021 | Published: 02 Feb, 2021

Citation: Ocampo MA, Capera C, Gonzalez MC, Salgado JM, Donoso W (2021) Angiomyolipoma with Intraluminal Tumor Thrombus into the Inferior Vena Cava: Insights and Treatment Alternatives. J Clin Case Stu 6(1): dx.doi.org/10.16966/2471-4925.212

Copyright: (c) 2021 Ocampo MA, et al. This is an open-access article distributed under the terms of the Creative Commons Attribution License, which permits unrestricted use, distribution, and reproduction in any medium, provided the original author and source are credited.

\begin{abstract}
Angiomyolipoma (AML) is a frequent benign renal tumor, corresponding to $0.3-3 \%$ of all renal masses. Eighty percent of $\mathrm{AML}$ occur sporadically; however, $10 \%$ have a hereditary component. We describe the case of a 41 -year-old female patient with left abdominal pain associated with emesis, diarrheal stools, and dysuria. Upon admission, a computed tomography scan showed a left renal mass with tumor thrombus extending into the left renal vein and inferior vena cava (IVC). The patient underwent an open left radical nephrectomy plus thrombectomy. At 6 months of follow-up, the patient is without tumor recurrence and asymptomatic.
\end{abstract}

Keywords: Renal cancer; Angiomyolipoma; Inferior vena cava; Tumor thrombus; Radical nephrectomy

\section{Introduction}

With the widespread use of diagnostic imaging, detection of incidental renal masses has increased. Many of these small tumors are benign, although a small portion represents malignant tumors and even with current imaging technology, it remains difficult to differentiate between them. The number of benign masses resected in the United States increased to $82 \%$ between 2000 and 2009 [1]. AML are one of the most frequently diagnosed benign renal tumors, their prevalence is estimated between $0.1-0.3 \%[2,3]$. These tumors occur more often in women, with a female to male ratio of 2:1 [2]. Despite that $80 \%$ of AML occurs sporadically, $10 \%$ have a hereditary component $[4,5]$. Tuberous sclerosis complex (TSC) is an autosomal dominant inherited disease, where $75 \%$ of patients may develop AML [4]

AML may be divided in two variants. The classic variant, characterized by being a triphasic tumor, composed of dysmorphic blood vessels, smooth muscle cells and fat $[1,2,4,6]$. There is also the epithelioid variant, that behaves more aggressively, which can present with metastasis, renal vein and vena cava tumoral thrombus invasion, with an estimated 3 year survival of 50\% [6,7]. The classic triad of AML symptoms includes flank pain, palpable mass, and hematurias, but when this is present, most have an advanced disease. Spontaneous retroperitoneal hemorrhage is the most frequent complication caused by tumor rupture, known as Wunderlich syndrome which canthreaten the patient's life [5]. We report an atypical case of classic pattern AML with tumor thrombus into the renal vein and the IVC.

\section{Case Presentation}

A 41-year-old female presented to the emergency department with pain in the left flank, diarrheal stools, emesis, and dysuria. She denies hematuria, weight loss or any other symptoms. Upon admission, an abdominal computed tomography (CT) was performed, which revealed a left renal mass, suggestive of angiomyolipoma, with tumor thrombus extending into the left renal vein and IVC (Figure 1). The patient underwent an open left radical nephrectomy plus thrombectomy of the IVC. At Surgical exploration, we found a left renal tumor that involved the middle and inferior pole of the kidney, measuring $15.2 \mathrm{~cm}$, with a tumor thrombus that extended from the renal vein to the IVC (Mayo II) (Figure 2). At microscopic histopathologic examination, findings were compatible with a renal AML, classic variant. Postoperative course was uneventful with the patient discharged on postoperative day 4 without complications. At 6-months follow-up, the patient is without relapse or radiological progression of the disease.

\section{Discussion}

Renal AMLs are benign tumors that originate from the renal parenchyma. They are mainly composed of adipose tissue, blood vessels and smooth muscle. Most of these lesions appear sporadically, however they can follow a hereditary pattern in up to $20 \%$ of patients with TSC $[4,6,8]$. AMLs were first described in 1900, but it wasn't until 1911 that Fische described its histopathological characteristics [2]. Spontaneous bleeding into the retroperitoneum or collecting system 


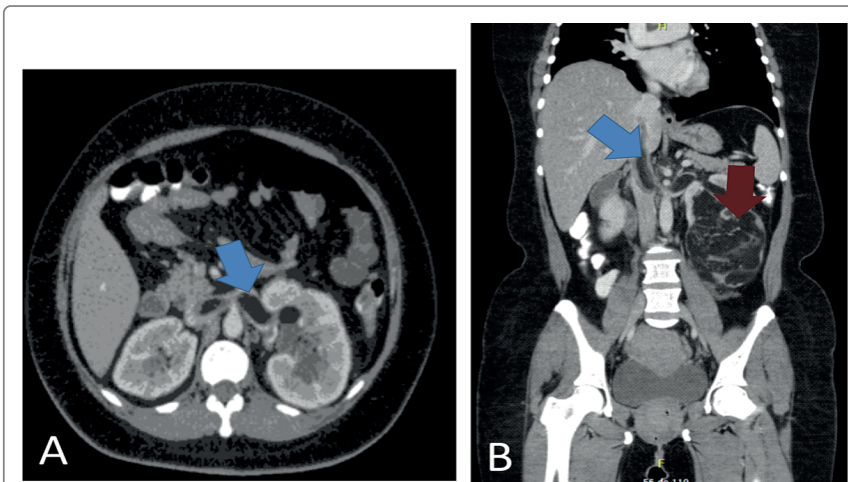

Figure 1: Abdominal Computerized Tomography (CT).

A. Tumoral thrombus extending through the inferior vena cava (blue arrow).

B. $15 \mathrm{~cm}$ Left renal mass with fat predominance, and $<15$ Hounsfield units (Red arrow).

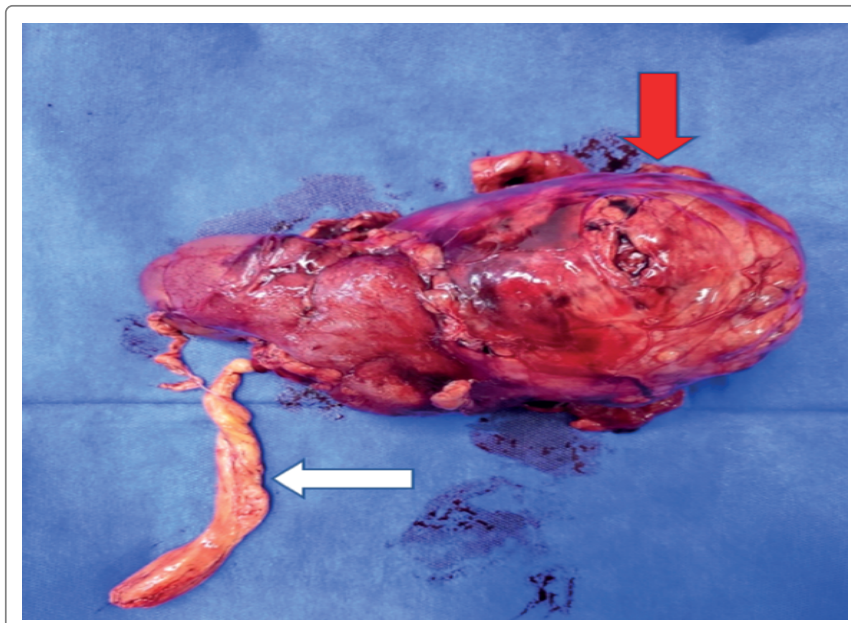

Figure 2: Macroscopic histopathological examination: Tumoral thrombus with macroscopic fat appearance (white arrow) and renal mass (red arrow).

is the most important complication of AML and it could be related to the vascular malformation. The risk of bleeding is proportional to the size of the mass and the size of the vascular malformation [8].

With the advent of diagnostic imaging, over $80 \%$ of these tumors are diagnosed as incidental cases with CT having a good diagnostic performance. The key radiological findings of these tumors are a hypodense lesion with an attenuation coefficient value of 15 or less Hounsfield units, which is suggestive of a macroscopic fatty component. In approximately $4-5 \%$ of cases there is no evidence of intralesional fat and these set of tumors, preferably called low-fat AML or fat-invisible AML, which can simulate renal cell carcinoma; and therefore, can be a diagnostic challenge for the urologist and the radiologist [6].

The treatment of renal AML has changed in the last four decades. In 1976, 93\% of patients were treated with nephrectomy. In 1986 Oesterling JE, et al. found that $82 \%$ of AMLs greater than $4 \mathrm{~cm}$ were symptomatic and, in these patients, surgical treatment should be considered, while observation should be offered to asymptomatic patients with masses less than $4 \mathrm{~cm}[4,9]$. Today there are several options for the treatment of AMLs, including active surveillance, selective vascular embolization, surgical management, and thermal ablation [8]. When surgical treatment is indicated, nephron sparing surgery is preferred. The association between tumor size and bleeding risk is not fully established; and therefore, the cut-off point of $4 \mathrm{~cm}$ to offer active treatment should be re-considered. The latest guidelines from the European Association of Urology (EAU) believe that active surveillance is the most appropriate option for AMLs. However, active treatment should be considered in cases where pain is persistent, tumor rupture occurs, or in cases of large tumor size. On the other hand, the disadvantages of selective embolization include a higher number of recurrences and the need for a second treatment; though, thermal ablation is an option that is used infrequently, due to insufficient data to date. Importantly, the patient must be comprehensively evaluated to provide the best active treatment option [10].

Until now there are a handful of reported cases of classic AML with tumor thrombus extending into the IVC. The first case dated back to 1982, reported by Kutche et al. [11]. It has been proposed that tumoral thrombi extension from an AML is not metastatic, but rather secondary to local tumor growth $[6,11,12]$. Nassib $K$, et al. reported that the presentation of tumor thrombi associated with AML is more frequently found in women, and most patients have a mean age at presentation of 50 years old. Most of the masses are right-sided tumors, and $62 \%$ of the patients present with symptoms at the time of diagnosis $[6,13]$. Radical nephrectomy with surgical thrombectomy has been proposed as the treatment of choice in patients with renal AML with IVC thrombus, even if they are asymptomatic at the time of diagnosis $[3,6,14]$. As in many cases the open approach is preferred when a thrombectomy is meant to be carried; although, there have been some cases treated with a laparoscopic or robotic-assisted approach, having the same oncological outcomes [14].

\section{Conclusion}

Renal AML with tumor thrombus extending into the IVC is rare. The treatment of choice is radical nephrectomy with tumor thrombectomy, which in most cases achieves good oncological results without neglecting the morbidity of the procedure.

\section{References}

1. Jinzaki M, Silverman SG, Akita H, Mikami S, Oya M (2017) Diagnosis of Renal Angiomyolipomas: Classic, Fat-Poor, and Epithelioid Types. Semin Ultrasound, CT MRI 38: 37-46.

2. Flum AS, Hamoui N, Said MA, Yang XJ, Casalino DD, et al. (2016) Update on the Diagnosis and Management of Renal Angiomyolipoma. J Urol 195: 834-846.

3. Gu J, Zeng L, Zeng H, Qin C, Wu Z (2018) A Rare Case of Renal Angiomyolipoma Involved both Inferior Vena Cava and Pulmonary Arteries. J Thorac Dis. 10: E166-E169.

4. Bhatt JR, Richard PO, Kim NS, Finelli A, Manickavachagam K, et al. (2016) Natural History of Renal Angiomyolipoma (AML): Most Patients with Large AMLs $>4 \mathrm{~cm}$ Can Be Offered Active Surveillance as an Initial Management Strategy. Euro Urol 70: 85-90.

5. Çalışkan S, Gümrükçü G, Özsoy E, Topaktas R, Öztürk Mi (2019) Renal Angiomyolipoma. Rev Assoc Med Bras 68: 977-981.

6. Heidar NFA, Degheili JA, Khauli RB, Saad GA (2020) A Large Bi-lobed Classic Renal Angiomyolipoma with Vena Caval Extension. Radiol Cas Repo 15: 353-361.

7. Seyam RM, Alkhudair WK, Kattan SA, Alotaibi MF, Alzahrani HM, et al. (2017) The Risks of Renal Angiomyolipoma: Reviewing the Evidence. J Kidney Cancer VHL 4: 13-25. 
8. Fernández-Pello $S$, Hora $M$, Kuusk $T$, Tahbaz $R$, Dabestani $S$, et al. (2020) Management of Sporadic Renal Angiomyolipomas: A Systematic Review of Available Evidence to Guide Recommendations from the European Association of Urology Renal Cell Carcinoma Guidelines Panel. Eur Urol Oncol 3: 57-72.

9. Ljungberg B, Bensalah K, Canfield S, Dabestani S, Hofmann F, et al. (2020) EAU Guidelines on Renal Cell Carcinoma: 2020 Update. Eur Urol 67: 913-924.

10. Oesterling JE, Fishman EK, Goldman SM, Marshall FF (1986) The Management of Renal Angiomyolipoma. J Urol 135: 1121-1124.

11. Shen G, Mao Q, Yang H, Wang C (2014) Aggressive Renal Angiomyolipoma with Vena Cava Extension: A Case Report and Literature Review. Oncol Lett 8: 1980-1982.
12. Lin WY, Chuang CK, Ng KF, Liao SK (2003) Renal Angiomyolipoma with Lymph Node Involvement: A Case Report and Literature Review. Chang Gung Med J 26: 607-610.

13. Fujiwara M, Kawamura N, Okuno T (2018) Preoperative Inferior Vena Cava Filter Implantation to Prevent Pulmonary Fat Embolism in a Patient Showing Renal Angiomyolipoma Extension into the Renal Vein: A Case Report and Literature Review. J Rural Med 13: 181-184.

14. Gutierrez-Zurimendi G, Albisu-Tristan A, Padilla-Nieva J, Acha-Pérez M, Urresola-Olabarrieta A, et al. (2020) Renal angiomyolipoma with invasion of the renal vein and inferior vena cava. Case report and literature review. Revis Urol Colombiana/Colomb Urol J 29: 099-102. 